\title{
Failure surface of quasi-periodic masonry by means of Statistically Equivalent Periodic Unit Cell Approach
}

\author{
N. Cavalagli · F. Cluni · V. Gusella
}

Received: date / Accepted: date

\begin{abstract}
In this paper a homogenization procedure for the estimation of the failure surface of a quasi-periodic masonry, based on a mean stresses approach through the analysis of the Statistically Equivalent Periodic Unit Cell (SEPUC), is shown. The mean stresses approach consists in the identification of critical states for the homogenized continuum by means of an overall failure criterion, function of the mean stress state of each constituent. These macroscopic tensors are evaluated in the elastic field. The SEPUC definition refers to a statistical criterion applied to a population of Periodic Unit Cells (PUCs) generated taking into account the geometrical features of the quasi-periodic texture; moreover it is validated on the basis of the homogenized elastic properties in terms of components and Frobenius norm of the elastic matrix. By a multi-objective optimization approach, the obtained results highlight that the proposed SEPUC can be used to estimate the failure surface.
\end{abstract}

Keywords Homogenization - Quasi-periodic masonry · Statistically Equivalent Periodic Unit Cell · Failure surface

\section{Introduction}

The mechanics of the masonry material is still today a matter of great interest for the scientific community due to both

\section{N. Cavalagli}

University of Perugia, Dep. Civil and Environmental Engineering, Via G. Duranti 93, 06125 Perugia Italy

E-mail: nicola.cavalagli@unipg.it

\section{F. Cluni}

University of Perugia, Dep. Civil and Environmental Engineering,

Via G. Duranti 93, 06125 Perugia Italy

E-mail: federico.cluni@unipg.it

V. Gusella

University of Perugia, Dep. Civil and Environmental Engineering,

Via G. Duranti 93, 06125 Perugia Italy

E-mail: vittorio.gusella@unipg.it the complexity of the material behaviour and the possibility to develop innovative strategies and approaches. In fact, the improvement of numerical methods in the study of solids mechanics and the increase of computational performance have favoured the development of advanced numerical strategies able to describe in a more comprehensive way the behaviour of complex non-homogeneous materials, such as masonry.

Dealing with heterogeneous materials, the homogenization techniques play a fundamental role in order to attain the effective properties of the homogenized continuum, taking into account the actual microstructure of the composite [1-4]. In this context, to consider the masonry material as a composite, made by inclusions (bricks or stones) and mortar matrix, has led to a fruitful development of analysis strategies based on the homogenization techniques. In the framework of the periodic texture, several methods have been proposed to obtain equivalent mechanical properties for masonry panels loaded in- and/or out-of-plane both in the elastic [5-8] and plastic field [9-15], also by using discrete approaches [16,17], interface modelling [18], multiscale and multigrid techniques [19-21] and micropolar material models [22-24].

The study of non-periodic texture requires different approaches owing to the impossibility of a direct definition and/or identification of the Representative Volume Element (RVE). Nevertheless, the application of several homogenization methods [25-27] has allowed to estimate the effective properties both in the elastic field [28-31] and at failure [3235].

However, must be also considered that, during the last years, the analysis of random composites has led to the development of statistical procedures aiming at the estimation of the homogenized elastic and plastic properties. Within the homogenization of random media, Ostoja-Starzewski [36] introduced the concept of Statistical Volume Element (SVE) 
dealing with the properties obtained at the mesoscale level, which can be described by the adjective apparent in opposition to the term effective attributed to the RVE. Other statistical approaches are aimed at the definition of a periodic cell characterized by a statistically representative microstructure [37]. Within this framework, several approaches can be found in literature, also distinguished by different acronyms assigned to the elementary cell. Among others some works are worthy of citing. Swaminathan and co-authors [38,39] have introduced the concept of the Statistically Equivalent Representative Volume Elements (SERVE) for the analysis of fiber-composites without and with damage. Zeman and Šejnoha [40] have proposed a statistical procedure based on the $n$-points probability function and lineal path to obtain the Statistically Equivalent Periodic Unit Cell (SEPUC) starting from actual images of the material microstructure. Moreover, Balzani and co-authors [41,42] have proposed a method for the construction of two- and three-dimensional Statistically Similar Representative Volume Elements (SSRVEs) by minimizing a least-square functional defined in terms of deviations of statistical measures describing the microstructure morphology and mechanical macroscopic entities. Trovalusci and co-authors [43] have recalled the concept of Statistical Volume Element (SEV) in the analysis of increasing portions size of random material in the searching for the effective properties.

The application of statistical procedures for the characterization of non-periodic masonry material is still an open problem. An interesting approach was given by Spence and co-authors [44], which have proposed a numerical procedure to generate random textures of chaotic masonry having the same statistical properties of an actual masonry texture. Lombardo and co-warkers [45] have performed a study for the elastic homogenization of chaotic masonry by means of perturbation methods. Šejnoha and co-authors [46] were the first to propose the application of the SEPUC concept to non-periodic masonry, in order to estimate homogenized properties and then applying the methodology to a case of study [47]. In this context, in a previous work [48] the Authors have proposed a geometrical method, based on the statistical analysis of the texture, to obtain the SEPUC of an actual quasi-periodic masonry panel. The definition of the SEPUC leads to the definition of a Statistically Equivalent Texture, namely a periodic texture which has the same statistical properties of the actual texture. The procedure is focused on the minimization of a function of random geometrical descriptors of the texture, such as the dimensions of the stones and of the mortar joints, which are randomly generated starting from probability density functions derived by the analysis of the actual texture. The term "quasi-periodic" is essentially referred to a masonry having the blocks arranged in almost continuous horizontal bed joints and random position of vertical joints, due to the random feature of the blocks width $[30,49]$. The criterion validation proposed in [48] was based on the error minimization of the overall elastic properties, namely the elastic matrix, calculated on the generated PUCs and on the actual masonry wall.

The aim of this work is to extend the procedure proposed by the Authors in [48] for the definition of the failure surface of a quasi-periodic masonry through the analysis of the SEPUC. It's well known that several approaches can be found in literature to estimate the failure surfaces of masonry material by means of phenomenological and theoretical models [50-55]. Dealing with non-periodic texture, differently from the procedures already available in literature, the proposed method allows to obtain the failure surface directly calculated on the SEPUC, in which the geometry of the cell, the inner texture and the periodicity conditions are defined. The essential concept of the numerical approach used lies in the identification of critical states by means of an overall failure criterion based on the strength values of the phases inside the heterogeneous solid assessed by their mean stress tensors. The analyses are performed in the elastic field with a generalized plane state formulation in axial deformation, so that the microcracks and the plastic strains commonly arisen before the global failure are considered in an overall manner. The effectiveness of this criterion in defining the failure surface of masonry material have been already proved by the Authors in previous works [35].

In particular, the novel contribution of this paper highlights that the statistical procedure used for the characterization of the SEPUC satisfies a multi-objective function based both to the homogenized elastic and at failure properties. This problem will be discussed referring to the concept of Pareto front, which considers a set of optimal solutions that can be taken into account.

\section{Problem statement}

In this paper the one-leaf masonry panel characterized by a quasi-periodic texture is studied. A quasi-periodic masonry can be considered characterized by a texture consisting of raws made by blocks with almost regular shape and nearly the same height. Nevertheless, the randomness of the blocks geometrical parameters determines that the rows may have different height. The heterogeneous solid is analysed through the generalized plane state formulation in axial strain, generally called in literature more simply generalized plane strain $(\mathrm{GPE})^{1}$, which can be considered a particular case of the generalized plane state [56]. The generalized plane state is a kinematic two-dimensional problem, provided with threedimensional components at the constitutive level, that can be

1 In the acronym GPE the letter " $E$ " is introduced as the latin form of the symbol " $\varepsilon$ ", generally used to designate strains. In this way possible misunderstandings derived by using the letter " $\mathrm{S}$ ", which could indicate both stress and strain, are avoided. 


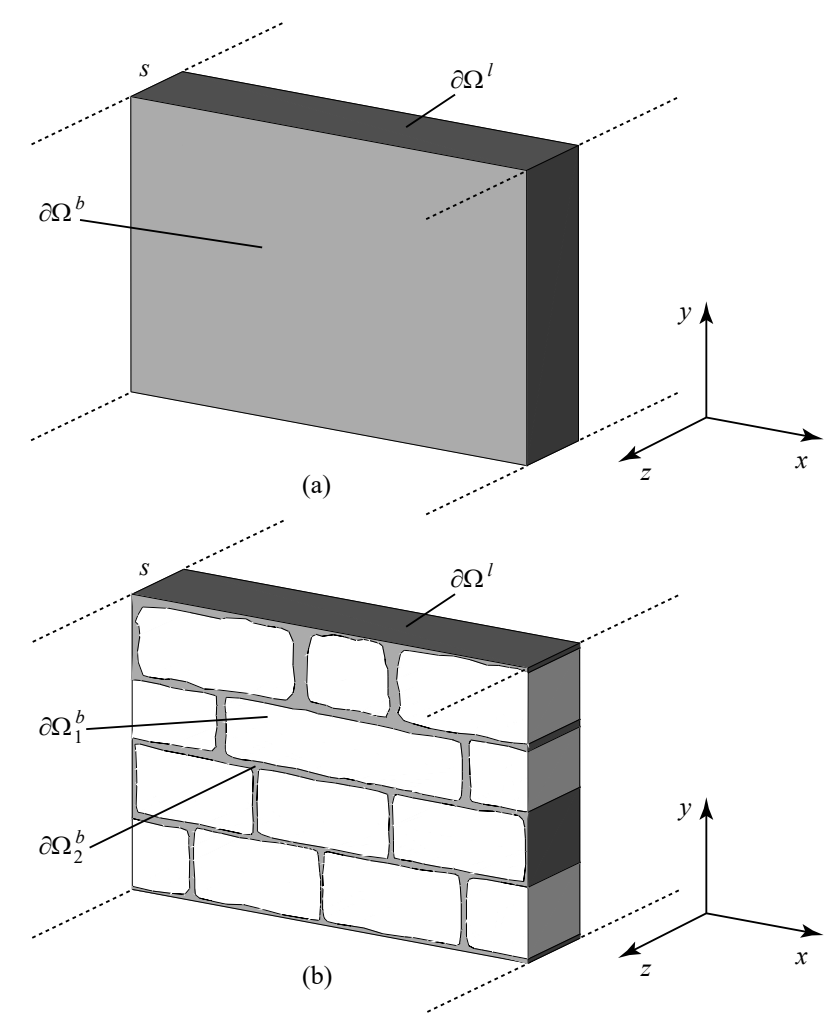

Fig. 1 Reference solid for the generalized plane state formulation in axial strain (GPE) for a homogeneous (a) and a bi-phase heterogeneous (b) material.

specialized in several plane problems. The effectiveness of such a formulation for the in-plane analysis of masonry panels at the mesoscale level has already been proved $[57,10]$, owing to the possibility of deriving out-of-plane stresses, often causes of critical states of failure, in a $2 \mathrm{D}$ problem. The reference solid is a cylinder $\Omega$ with finite length $s$ placed in a $\mathbb{R}^{3}$ Euclidean space, referred to $(x, y, z)$ coordinate system, being $z$ the longitudinal axis of the cylinder (Fig. 1). In the following both the cases of homogeneous (Fig. 1(a)) and multi-phase solid (Fig. 1(b)) will be taken into account. The latter, in the more general case, is made up of $n$ phases whose distribution varies randomly in the $(x, y)$ plane, but not in the $z$ direction. As indicated in Fig. $1, \partial \Omega^{l}$ and $\partial \Omega^{b}$ denote the lateral and the base surfaces of the cylinder respectively. A linear elastic and isotropic behaviour is assumed for both the phases.

The basic hypothesis is that the stress and strain tensors are independent from the $z$ direction as

$$
\begin{aligned}
& \sigma=\sigma(x, y) \\
& \varepsilon=\varepsilon(x, y) .
\end{aligned}
$$

Let us consider the displacement vector $\mathbf{u}(u, v, w)$. The equations (1) and (2) imply, through the compatibility conditions and kinematic equations of elasticity, that the general form of the displacements field is

$$
\begin{aligned}
& u=-\frac{A}{2} z^{2}+D y z+F z+f_{1}(x, y) \\
& v=-\frac{B}{2} z^{2}-D x z+H z+f_{2}(x, y) \\
& w=(A x+B y+C) z+f_{3}(x, y) .
\end{aligned}
$$

where the constants $A, B, C, D, F, H$ and the functions $f_{1}, f_{2}$ and $f_{3}$ depend on the boundary conditions imposed on the basis and determine different types of plane problems. The interested reader is invited to see $[58,56,35]$ for the details of the elastic formulation.

The case of generalized plane state in axial strain (GPE) is characterized by the following conditions $A=B=D=0$, $F=H=0$ and $f_{3}=0$. These assumptions determine the absence of the tangential components in the longitudinal direction for the stress and strain tensors and, at the same time, constraint the generic cross section of the solid to be plane and normal to the $z$ direction. The displacements field becomes

$$
\begin{aligned}
& u=f_{1}(x, y) \\
& v=f_{2}(x, y) \\
& w=C z
\end{aligned}
$$

The solving equations of the elastic problem are reduced to 10 (2 equilibrium equations, 4 kinematic conditions and 4 constitutive relations) with 11 unknowns (stress and strain components and displacements field). The further equation is obtained by the overall equilibrium condition in the $z$ direction. In the case of the heterogeneous solid, the equilibrium equation can be written as a summation over the $n$ phases

$$
\sum_{i=1}^{n} \int_{\partial \Omega_{i}^{b}} \sigma_{z z}^{(i)} \mathrm{d} x \mathrm{~d} y=0
$$

where $\sigma_{z z}^{(i)}$ and $\partial \Omega_{i}^{b}$ indicate the out-of-plane stresses and the area of the $i$ th phase, so that

$$
\sum_{i=1}^{n} \partial \Omega_{i}^{b}=\partial \Omega^{b}
$$

Using the (9) together with the constitutive relations and the strain condition

$\varepsilon_{z z}=C$

derived by the equation (8), the value of the constant $C$ is obtained by

$C=-\frac{\sum_{i=1}^{n} \frac{\lambda_{i}}{2\left(\lambda_{i}+\mu_{i}\right)} \int_{\partial \Omega_{i}^{b}}\left(\sigma_{x x}^{(i)}+\sigma_{y y}^{(i)}\right) \mathrm{d} x \mathrm{~d} y}{\sum_{i=1}^{n} \frac{\mu_{i}\left(3 \lambda_{i}+2 \mu_{i}\right)}{\lambda_{i}+\mu_{i}} \partial \Omega_{i}^{b}}$.

in which $\lambda_{i}$ and $\mu_{i}$ are Lamè elastic parameters of the $i$ th phase. 

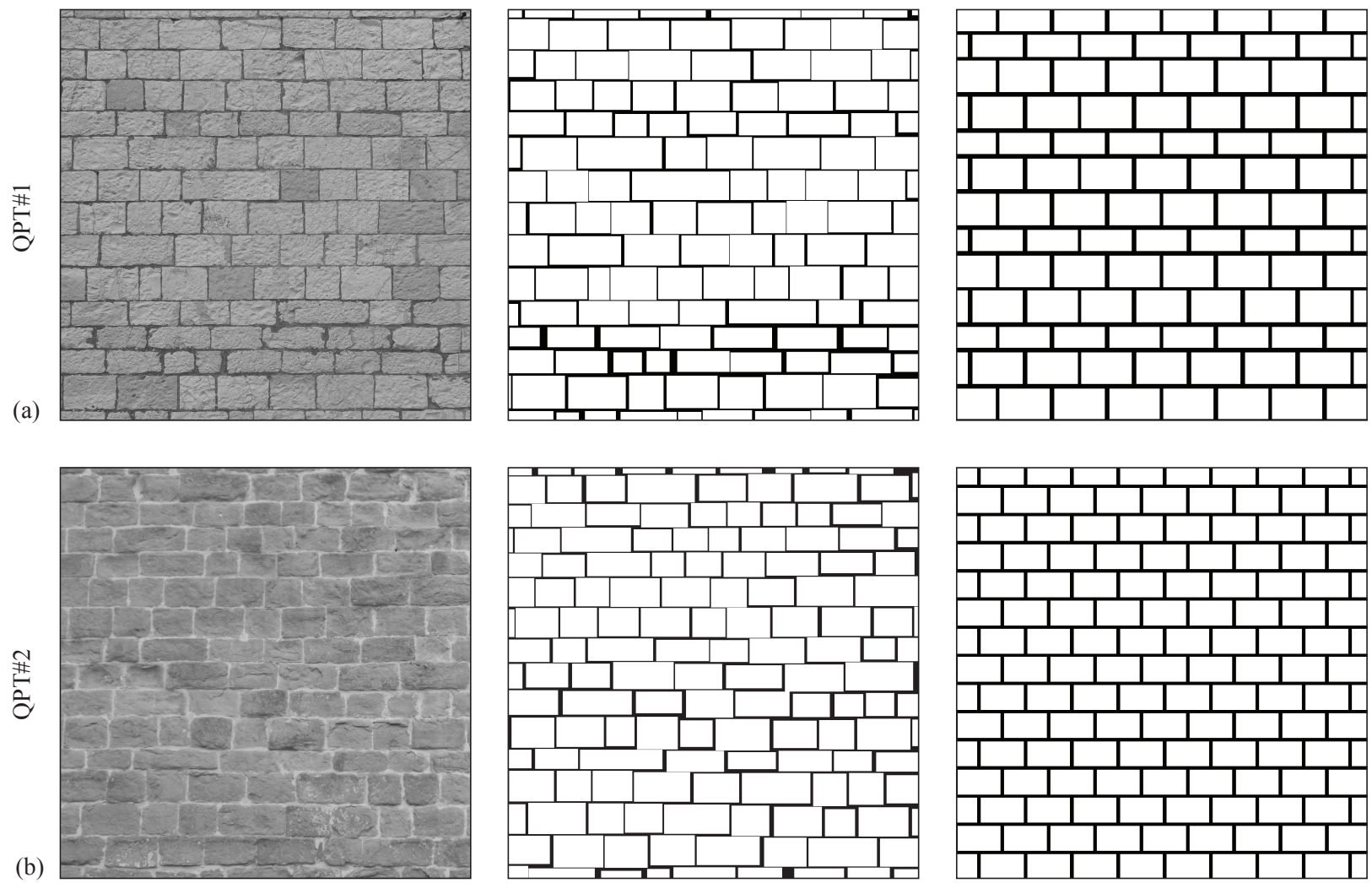

Fig. 2 Cases of study of actual Quasi-Periodic Textures (QPT) used in the procedure which leads to the Statistically Equivalent Textures [48].

Unlike the most commonly used plane stress state, this kind of constitutive formulation allows to take into account the out-of-plane behaviour of the masonry material. In particular, the presence of the $\sigma_{z z}$ stresses assumes a relevant role in the modelling of some failure mechanisms, which occur, for example, in biaxial stress state of a masonry panel.

\section{Statistically Equivalent Periodic Unit Cell (SEPUC)}

As stated in the Introduction, the texture of a masonry wall can be defined as "quasi-periodic" if the inclusions, bricks or stones, are quasi regular and arranged in horizontal rows. Under this hypothesis, a Periodic Unit Cell (PUC), having geometrical properties statistically equivalent to the actual quasi-periodic texture, can be easily attained by means of a statistical analysis of predefined geometrical parameters.

In a previous work [48], the Authors have established a mean stress based criterion to obtain the Statistically Equivalent Periodic Unit Cell (SEPUC) complying with the elastic response of the actual walls with quasi-periodic textures. In the following, the applications already presented in that work are briefly recalled and, in the following, more in-deep studied as far as it concerns the elastic properties and then analysed at the elastic limit with the definition of the failure surfaces.
Let us consider the two wall masonry samples shown in Fig. 2, where a schematic process of analysis, based on automatic procedures of digital image processing, which allows the passage from an actual quasi-periodic texture to the Statistically Equivalent Texture (SET) is illustrated for both of them.

The Quasi-Periodic Textures \#1 and \#2 (QPT\#1 and QPT\#2 of Fig. 2) consist of stones with different dimensions, but of roughly rectangular shape, connected by mortar joints. The sample is $2000 \mathrm{~mm}$ in length $2000 \mathrm{~mm}$ in height. The gray scale image has been elaborated with digital image tools to obtain the black and white image, with a consistent separation of phases. In order to estimate the SEPUC, the stones have been regularized to an exact rectangular shape, as shown in the central sketches of Fig. 2. In this passage, it has been assumed that the original stone and the regularized one have the same area and the same ratio of principal moment of inertia. This allowed to estimate the statistical distribution of the following geometrical descriptors: width of the stones, height of the stones, thickness of bed and head mortar joints.

The statistical analysis have pointed out that in the case of QPT\#1 the geometrical descriptors are characterized by unimodal distributions with the exception of the height of the stones, which has a bimodal distribution. Differently, the case of QPT\#2 has all the geometrical descriptors with uni- 
modal distributions. These features have affected the SEPUC morphology for both the cases. The interested reader is invited to see [48]. In particular, for QPT\#1 the results was a running bond pattern, but using stones of two different heights (with a ratio grater/smaller of 2/1), while for QPT\#2 a classic running bond pattern was obtained.

Thereafter, the SEPUC was determined, by generating 1000 cells (samples) $\left(\Psi^{1}, \ldots, \Psi^{1000}\right)$ with random dimensions, according to geometrical descriptors distributions [48]. These random samples define Periodic Unit Cells (PUCs)which, however, could not be representative of the actual texture, even if the geometrical parameters are generated from probability density functions extracted from the actual texture itself. The SEPUC is chosen among the 1000 PUCs adopting a criterion which minimizes an error function defined on the $q$ geometrical descriptors $\mathscr{X}_{k}$ and the concentration ratio $\chi$ of a phase

$\mathscr{F}\left(\Psi^{i}\right)=\frac{1}{q+1}\left(\sum_{k=1}^{q} \frac{\left|\mathscr{X}_{k}^{i}-\mathscr{X}_{k}^{W}\right|}{\mathscr{X}_{k}^{W}}+\frac{\left|\chi_{1}^{i}-\check{\chi}_{1}^{W}\right|}{\check{\chi}_{1}^{W}}\right)$

In particular were used five geometrical descriptors $\mathscr{X}_{i}$ for QPT\#1 (width and two heights of stones, thickness of bed and head joints), and four for QPT\#2 (width and height of stones, thickness of bed and head joints). The concentration ratio $\chi_{1}^{i}$ is defined as the percentage of the area of masonry wall occupied by the blocks. The index $i$ refer the $i$ th of the 1000 PUCs and $W$ to the entire wall (in terms of mean values).

The identified SEPUCs (SEPUC\#1 and SEPUC\#2 for QPT\#1 and QPT\#2 respectively) have the dimensions shown in (Fig. 3). The resulting SETs of the considered masonry walls obtained with the SEPUCs are illustrated on the right side of Fig. 2 as the result of the analysis. Details on the procedure are reported in [48].

In the following, after the description of the periodic boundary conditions applied, the homogenized elastic properties and failure surfaces of the SEPUCs are reported. For the sake of clarity, the results obtained for QPT\#1 will be shown in the main text of the paper, while QPT\#2 has been included in the App. A. Moreover, in order to validate the procedure and to compare the results, a more simple morphology of the SEPUC\#1 has been analysed, considering a single block arranged in a classic running bond texture. In this way the bimodal property of the height stones distribution is not considered and the generation of 1000 PUCs has been made taking into account only four geometrical descriptors, as in the case of QPT\#2. The results derived by the application of the criterion (13) identifies a periodic cell with the following values geometrical descriptors: stones width and height of $23.1 \mathrm{~cm}$ and $14.6 \mathrm{~cm}$ respectively, and thickness of bed and head joint of $1.3 \mathrm{~cm}$ and $1.4 \mathrm{~cm}$ respectively. This cell will be called SEPUC\#1-RB.

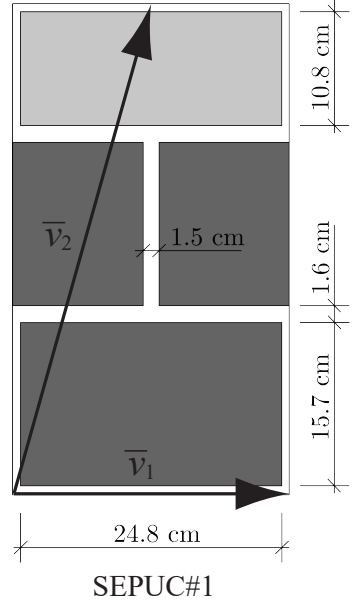

(a)

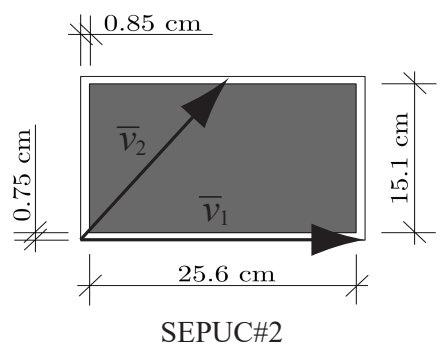

(b)
Fig. 3 Statistically Equivalent Periodic Unit Cells (SEPUC\#1 (a) and SEPUC\#2 (b)) with their translation vector system $\bar{v}_{1} \bar{v}_{2}$ obtained for the cases of QPT\#1 and QPT\#2 [48].

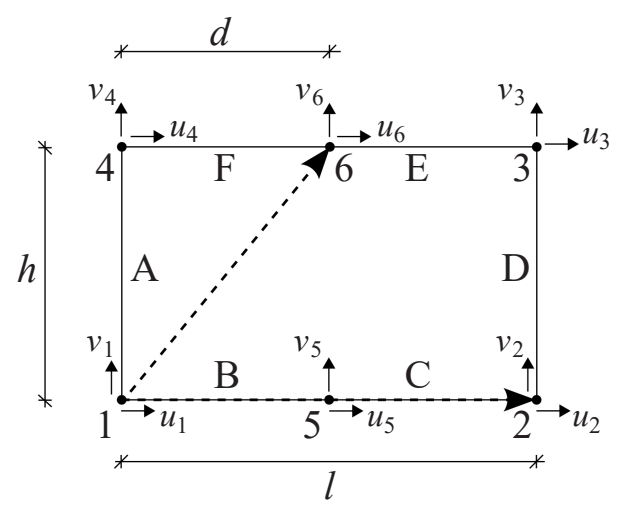

Fig. 4 Translation system and periodic reference system of the unit cell.

\section{The Unit Cell and the periodic boundary conditions}

The homogenized mechanical properties of a masonry panel with periodic texture can be directly obtained through the imposition of periodic conditions at the boundaries of the cell. These boundary conditions must assure the periodicity of the deformations and the anti-periodicity of the stresses in the cell [5]. It's well known that the definition of the periodic boundary conditions depends on the shape of the unit cell and on the reference system to which the translation vectors are based.

Taking into account non-periodic masonry textures, it is conceivable to assess that a periodic pattern can be always given by a rectangular unit cell composed by six nodes, three of which are necessary to identify the translation vectors base (nodes 1, 2 and 6 called "controlling points"), and six sides on which the periodicity conditions are defined (Fig. 4). The above consideration is valid for $0<d<l$, otherwise a simple four-nodes rectangular cell with orthogonal 
translation vectors is sufficient to describe the periodic texture, e.g. for the classic stack bond pattern. Owing to the randomness of the masonry pattern studied in this work, this case has not been considered. The periodic cell of Fig. 4, having area $\Lambda$, is defined in the plane of the generic wall, i.e. the $(x, y)$ plane. According to the formulation described in the previous section, all the points are characterized by $u$ and $v$ displacement functions, in $x$ and $y$ direction respectively. The geometrical properties and the distribution of the inclusions inside the cell depends by the type of texture of the case study.

The definition of specific periodic boundary conditions in terms of forces and/or displacements imposed on three of the six degrees of freedom of the controlling points allows to obtain macroscopic states of stresses $\langle\sigma\rangle$ and strains $\langle\varepsilon\rangle$ for the estimation of the homogenized properties. In particular $\langle\sigma\rangle$ and $\langle\varepsilon\rangle$ are obtained by means of

$\langle\sigma\rangle=\frac{1}{\Lambda} \int_{\Lambda} \sigma(x, y) \mathrm{d} \Lambda$

$\langle\varepsilon\rangle=\frac{1}{\Lambda} \int_{\Lambda} \varepsilon(x, y) \mathrm{d} \Lambda$

where $\sigma(x, y)$ and $\varepsilon(x, y)$ are the local values of the stress and strain tensors. The elastic tensor $\mathbf{C}$ relates the macroscopic stress tensor with the macroscopic strain tensor as

$\langle\sigma\rangle=\mathbf{C}\langle\varepsilon\rangle$

and in its explicit form as

$\left\{\begin{array}{l}\left\langle\sigma_{x x}\right\rangle \\ \left\langle\sigma_{y y}\right\rangle \\ \left\langle\sigma_{x y}\right\rangle\end{array}\right\}=\left[\begin{array}{ccc}C_{11} & C_{12} & 0 \\ C_{21} & C_{22} & 0 \\ 0 & 0 & C_{33}\end{array}\right]\left\{\begin{array}{l}\left\langle\varepsilon_{x x}\right\rangle \\ \left\langle\varepsilon_{y y}\right\rangle \\ \left\langle\varepsilon_{x y}\right\rangle\end{array}\right\}$

Following the mathematical approach of [7], $u_{2}, v_{2}$ and $v_{6}$, are the master d.o.f. in which prescribed boundary conditions are applied. The other d.o.f. of the controlling points are restrained or constrained in order to avoid rigid motions of the cell

$u_{1}=v_{1}=0$

$u_{6}=\frac{d}{l} u_{2}+\frac{h}{l} v_{2}=\frac{1}{2} u_{2}+\frac{h}{l} v_{2}$

in which $l, h$ and $d$ are the geometric parameters describing the dimensions of the cell and of the translation vector (Fig. 4). In the second equation of (18), the relation $d=l / 2$ for the considered cases has been included. Considering the points belonging to the six sides of the cell $A, \ldots, F$, the periodicity conditions in vector format are:

$\mathbf{u}_{D}-\mathbf{u}_{A}=\mathbf{u}_{2}-\mathbf{u}_{1}$

$\mathbf{u}_{E}-\mathbf{u}_{B}=\mathbf{u}_{6}-\mathbf{u}_{1}$

$\mathbf{u}_{F}-\mathbf{u}_{C}=\mathbf{u}_{6}-\mathbf{u}_{2}$ which can be expressed in terms of single components considering the boundary conditions (18)

$u_{D}-u_{A}-u_{2}=0$

$v_{D}-v_{A}-v_{2}=0$

$u_{E}-u_{B}-\frac{1}{2} u_{2}+\frac{h}{l} v_{2}=0$

$v_{E}-v_{B}-v_{6}=0$

$u_{F}-u_{C}-\frac{1}{2} u_{2}+\frac{h}{l} v_{2}=0$

$v_{F}-v_{C}-v_{6}+v_{2}=0$

Specific values of boundary conditions assigned at the master d.o.f. can determine predefined macroscopic stress $\langle\sigma\rangle^{0}$ or strain $\langle\varepsilon\rangle^{0}$ tensors into the cell. Considering the following three elementary tensors

$\mathbf{I}_{1}=\left[\begin{array}{ll}1 & 0 \\ 0 & 0\end{array}\right], \quad \mathbf{I}_{2}=\left[\begin{array}{ll}0 & 0 \\ 0 & 1\end{array}\right], \quad \mathbf{I}_{3}=\left[\begin{array}{ll}0 & 1 \\ 1 & 0\end{array}\right]$

the base stress tensors are obtained by means of a strain approach, i.e. by analyzing the three basic cases $\langle\varepsilon\rangle_{\xi}^{0}=\mathbf{I}_{\xi}$, $\xi=1,2,3$ through the relations

$\left\{\begin{array}{l}u_{2_{\xi}}=l\left\langle\varepsilon_{x x}\right\rangle_{\xi}^{0} \\ v_{2_{\xi}}=l\left\langle\varepsilon_{y y}\right\rangle_{\xi}^{0} \\ v_{6_{\xi}}=h\left\langle\varepsilon_{y y}\right\rangle_{\xi}^{0}+l\left\langle\varepsilon_{x y}\right\rangle_{\xi}^{0}\end{array}\right.$

\section{Elastic properties of the SEPUC}

The criterion (13) explained in [48], which takes into account the deviation from the mean values of the geometrical descriptors and of the concentration ratio, has been recognised as the best representative, among others proposals, on the basis of the homogenized elastic properties of the optimal cell expressed only in terms of the Frobenius norm. In this section, a more detailed sight of the elastic matrices of the PUCs is shown in order to carry out a better evaluation of the multi-objective analysis, which takes into account homogenized properties in the elastic range and at failure.

The homogenized elastic matrix of each PUC $\left(\mathbf{C}^{\mathrm{PUC}}\right)$ has been obtained by means of the direct method, applying the three cases of displacement boundary conditions defined by (22). The components of the elastic matrices obtained on the PUCs have been compared with those estimated on the entire masonry wall (Fig. 2) through the approach proposed by [28], based on Hill's definition of the RVE [1], by applying uniform boundary conditions expressed in terms of forces and displacements. Two types of uniform boundary conditions have been applied on the masonry wall: 


\begin{tabular}{cccccc}
\hline $\mathrm{ij}$ & $\mathbf{C}_{\varepsilon}^{\text {app }}$ & $\mathbf{C}_{\sigma}^{\text {app }}$ & $\mathbf{C}^{\text {eff }}$ & $\mathbf{C}^{\text {SEPUC\#1 }}$ & $\mathbf{C}^{\text {SEPUC\#1-RB }}$ \\
\hline 11 & 5590.0 & 5554.6 & 5572.3 & $5581.5(+0.16 \%)$ & $5646.7(+1.33 \%)$ \\
22 & 5194.6 & 5168.8 & 5181.7 & $5208.6(+0.52 \%)$ & $5412.4(+4.45 \%)$ \\
33 & 1991.8 & 1972.7 & 1982.2 & $2003.5(+1.07 \%)$ & $2078.4(+4.85 \%)$ \\
12 & 1006.9 & 1006.0 & 1006.5 & $855.8(-14.97 \%)$ & $881.9(-12.38 \%)$ \\
\hline
\end{tabular}

Table 1 QPT\#1 results. Components of the elastic tensor of masonry wall evaluated by the application of natural $\left(\mathbf{C}_{\sigma}^{\text {app }}\right)$ and essential $\left(\mathbf{C}_{\varepsilon}^{\text {app }}\right)$

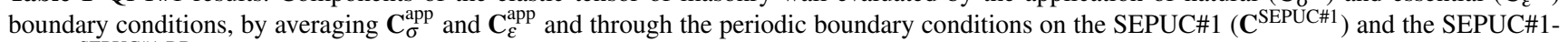
$\mathrm{RB}\left(\mathbf{C}^{\text {SEPUC\#1-RB }}\right)$. (values in MPa)

- natural boundary conditions, in which a self-balanced load system is applied

$$
\mathbf{t}(\mathbf{x})=\langle\sigma\rangle^{0} \mathbf{n}(\mathbf{x}) \quad \forall \mathbf{x} \in \partial \Lambda
$$

where $\mathbf{n}(\mathbf{x})=\left(n_{x}(\mathbf{x}), n_{y}(\mathbf{x})\right)^{T}$ is the versor of the normal to the boundary of the portion, $\partial \Lambda$, at $\mathbf{x}$ and $\langle\sigma\rangle^{0}$ is a matrix of order two;

- essential boundary conditions, in which a displacement field is imposed

$$
\mathbf{u}(\mathbf{x})=\langle\varepsilon\rangle^{0} \mathbf{x} \quad \forall \mathbf{x} \in \partial \Lambda
$$

where $\langle\varepsilon\rangle^{0}$ is a matrix of order two.

Considering the three elementary tensors previously introduced (21), in the case of natural boundary conditions the forces are given by

$$
\begin{array}{lll}
\langle\sigma\rangle_{1}^{0}=\mathbf{I}_{1} & \Rightarrow & \mathbf{t}(\mathbf{x})=\left(\begin{array}{c}
n_{x}(\mathbf{x}) \\
0
\end{array}\right) \\
\langle\sigma\rangle_{2}^{0}=\mathbf{I}_{2} & \Rightarrow & \mathbf{t}(\mathbf{x})=\left(\begin{array}{c}
0 \\
n_{y}(\mathbf{x})
\end{array}\right) \\
\langle\sigma\rangle_{3}^{0}=\mathbf{I}_{3} & \Rightarrow & \mathbf{t}(\mathbf{x})=\left(\begin{array}{l}
n_{y}(\mathbf{x}) \\
n_{x}(\mathbf{x})
\end{array}\right)
\end{array}
$$

while for essential boundary conditions the displacements are given by

$$
\begin{array}{llll}
\langle\varepsilon\rangle_{1}^{0}=\mathbf{I}_{1} & \Rightarrow & \mathbf{u}(\mathbf{x})=\left(\begin{array}{l}
x \\
0
\end{array}\right) \\
\langle\varepsilon\rangle_{2}^{0}=\mathbf{I}_{2} & \Rightarrow & \mathbf{u}(\mathbf{x})=\left(\begin{array}{l}
0 \\
y
\end{array}\right) \\
\langle\varepsilon\rangle_{3}^{0}=\mathbf{I}_{3} & \Rightarrow & \mathbf{u}(\mathbf{x})=\left(\begin{array}{l}
y \\
x
\end{array}\right) .
\end{array}
$$

For both the cases QPT\#1 and QPT\#2, the elastic properties assigned to the blocks $\left(E_{b} ; v_{b}\right)$ and $\operatorname{mortar}\left(E_{m} ; v_{m}\right)$ are the same used in [35], i.e. $E_{b}=6740 \mathrm{MPa} ; v_{b}=0.167 ; E_{m}=$ $1700 \mathrm{MPa}$ and $v_{m}=0.2$. Considering the case of QPT\#1, in Tab. 1 the following results in terms of elastic matrices components are summarized $[35,48]$ : the apparent values by the application of natural $\left(\mathbf{C}_{\sigma}^{\text {app }}\right)$ and essential $\left(\mathbf{C}_{\mathcal{E}}^{\text {app }}\right)$ boundary conditions; the effective values $\left(\mathbf{C}^{\text {eff }}\right)$ given by averaging $\mathbf{C}_{\sigma}^{\text {app }}$ and $\mathbf{C}_{\varepsilon}^{\text {app }}$; homogenized components $\left(\mathbf{C}^{\text {SEPUC\#1 }}\right)$ obtained by the application of periodic boundary conditions on the SEPUC\#1 identified by the objective function (13); homogenized components ( $\mathbf{C}^{\text {SEPUC\#1-RB }}$ ) obtained by the application of periodic boundary conditions on the SEPUC\#1$\mathrm{RB}$ constituted by a single block arranged in the standard running bond pattern. In Fig. 5 these results are compared with the values related to generated PUCs. The orange band highlights the zone limited by the lower and upper bounds, i.e. the values given by the application of natural and essential boundary conditions, the blue circle identifies the SEPUC\#1 and the grey line indicates the value obtained on the SEPUC\#1-RB. It should be noted that the elastic matrix components of the SEPUC\#1 are very close to the bounds except for $C_{12}$. Nevertheless, the most part of the points cloud $C_{12}^{\mathrm{PUC}}$ are distant from lower and upper bounds due to the regularity of the inclusions which characterizes the generated PUC versus the actual texture. It is reasonable to be expected that after the regularization process (central image of Fig. 2(a)) the shear stiffness of the wall is lower than the actual, due to the reduced interlocking effect between the inclusions derived by the regularization of the blocks shape.

An overall representation of the elastic matrices $\mathbf{C}^{\mathrm{PUC}}$ for the comparison with $\mathbf{C}^{\text {app }}$ and $\mathbf{C}^{\text {eff }}$ can be given by the Frobenius norm $\|\mathbf{C}\|$ directly applied on the elastic matrices as [48]

$$
\|\mathbf{C}\|=\sqrt{\sum_{i=1}^{3} \sum_{j=1}^{3} C_{i j}^{2}}
$$

In Fig. 6(a) the points cloud of the Frobenius norm evaluated in each PUC is compared with the values obtained for $\mathbf{C}_{\sigma}^{\text {app }}$ and $\mathbf{C}_{\varepsilon}^{\text {app }}$.

Moreover, a novel overall measure is proposed: the difference between the elastic matrices of the PUCs $\left(\mathbf{C}^{\mathrm{PUC}}\right)$ and the effective one $\left(\mathbf{C}^{\text {eff }}\right)$ evaluated as the Frobenius norm of the components' differences

$\left\|\Delta \mathbf{C}^{\mathrm{PUC}}\right\|=\sqrt{\sum_{i=1}^{3} \sum_{j=1}^{3}\left(C_{i j}^{\mathrm{PUC}}-C_{i j}^{\mathrm{eff}}\right)^{2}}$

In Fig. 6(b) the values of $\left\|\Delta \mathbf{C}^{\mathrm{PUC}}\right\|$ normalized with respect to $\left\|\mathbf{C}^{\text {eff }}\right\|$ are represented.

The elastic analyses have been carried out also to the case of QPT\#2. See Appendix A for the results. 

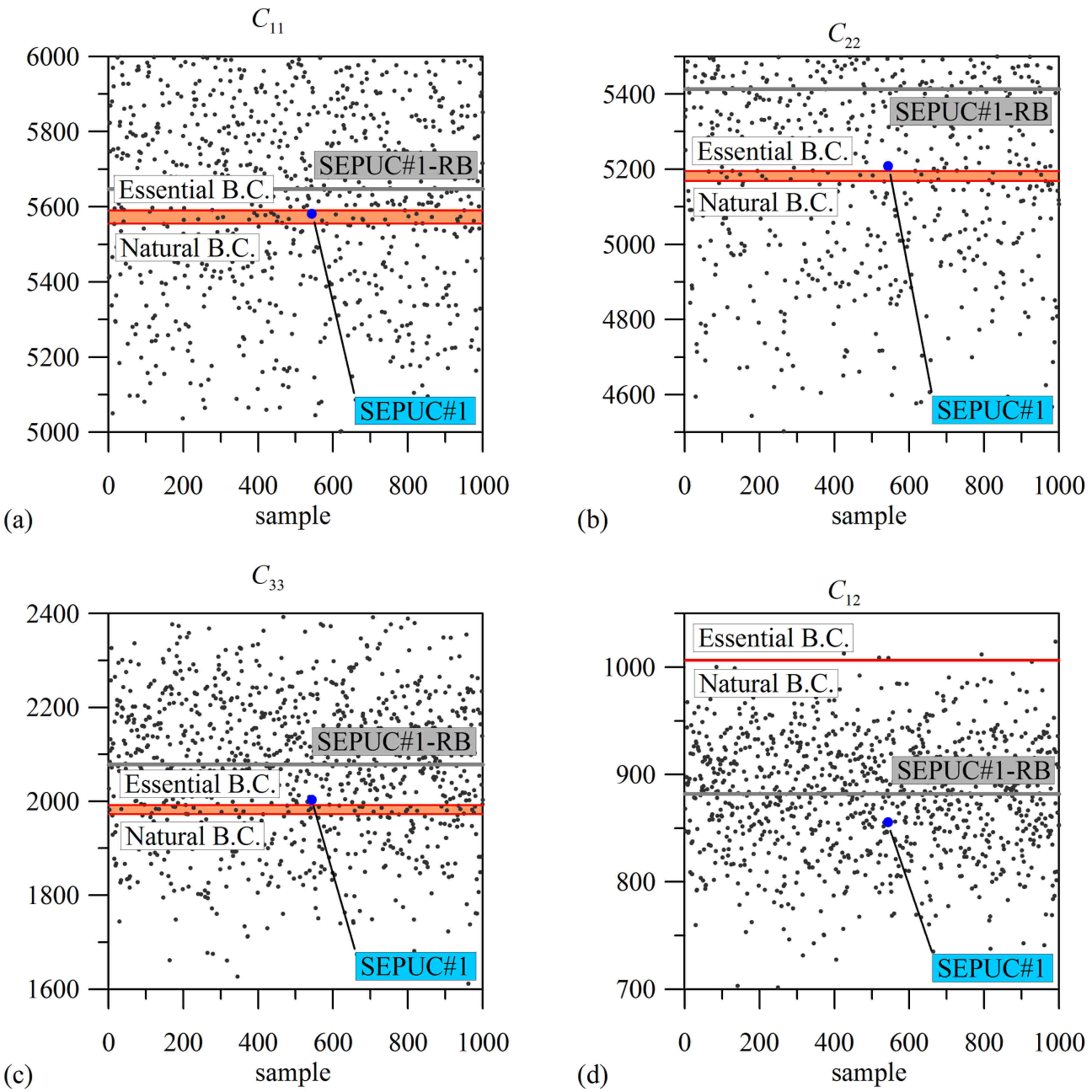

Fig. 5 QPT\#1 results. Comparison between the elastic matrix components (values in MPa) evaluated for each PUC, through the application of periodic boundary conditions (black points), and for the QPT\#1, through the application of essential and natural boundary conditions (red lines). The blue point identifies the SEPUC\#1 given by the objective function (13). The grey lines indicate the elastic matrix components obtained on the SEPUC\#1-RB, constituted by a single block arranged in the standard running bond pattern.
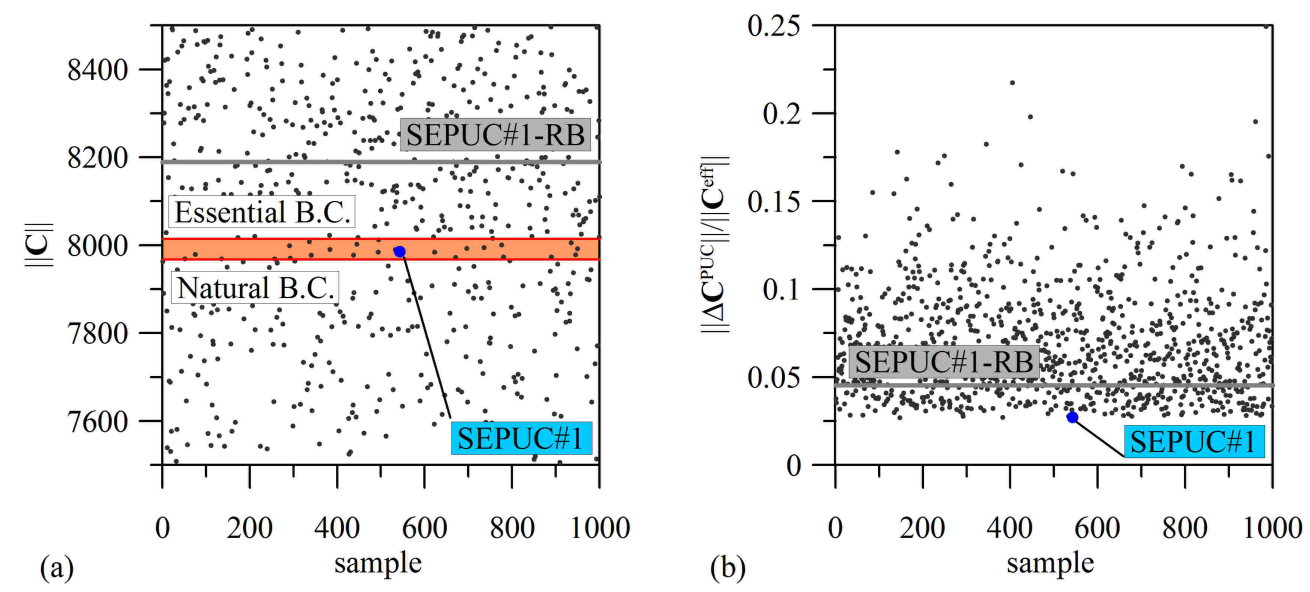

Fig. 6 QPT\#1 results. (a) Comparison between the Frobenius norm of the elastic matrix evaluated for each PUC, thorugh the application of periodic boundary conditions (black points), and for the QPT\#1, through the application of essential and natural boundary conditions (red lines). (b) Error estimation $\left\|\Delta \mathbf{C}^{\mathrm{PUC}}\right\|$ evaluated for each PUC normalized with respect to the effective elastic matrix norm $\left\|\mathbf{C}^{\text {eff }}\right\|$. The blue point identifies the SEPUC\#1 and the grey line indicates the value obtained for the SEPUC\#1-RB. 


\section{Failure surface of the SEPUC}

\subsection{Overall and local failure criteria}

The definition of a unique failure criterion for a composite material, such as masonry in this case, must take into account the mechanical features of each constituent, because, generally, all of them participate to the overall strength with own properties.

The method used in this paper is based on the work of Dvorak and Bahei-El-Din [59] and consists of considering that the overall failure of the heterogeneous solid is reached when, by increasing the values of the boundary loads (or alternatively of the boundary displacements), the mean stress tensor of one of the phases satisfies its own failure condition. In other words, working the procedure in the elastic field, the local evolution of non-linearity effects developed before the overall failure are taken into account in mean sense. On the other hand, the method, even if approximated, allows to perform elastic analyses in generalized plane state in axial deformation with significant computational advantages and, at the same time, adequate results [35].

The linearity of the approach and the plane kinematic definition of the problem have allowed to analyse a base of the macroscopic stress tensors, which then have been combined through the superposition principle.

Being $\langle\sigma\rangle_{1},\langle\sigma\rangle_{2}$ and $\langle\sigma\rangle_{3}$ the macroscopic stress tensors related to the cases $\langle\varepsilon\rangle_{1}^{0},\langle\varepsilon\rangle_{2}^{0}$ and $\langle\varepsilon\rangle_{3}^{0}$ respectively, the general state of stress can be given by means of the coefficients $a, b, c$ in the expression

$\langle\sigma\rangle=a\langle\sigma\rangle_{1}+b\langle\sigma\rangle_{2}+c\langle\sigma\rangle_{3}$

The overall failure condition of the heterogeneous material in the macroscopic stress space $\langle\sigma\rangle$ is defined as [35]

$F(\langle\sigma\rangle)=\inf \left\{\langle\sigma\rangle \in \mathbb{R}^{(2 \times 2)} \mid f^{(i)}\left(\mathbf{K}^{(i)}\langle\sigma\rangle\right)=0, i=1,2\right\}$

where $f^{(i)}$ and $\mathbf{K}^{(i)}$ are the failure criterion and the elastic matrix concentration of the phase $i$.

The local failure criterion used for both the constituents, blocks and mortar, is a concrete model proposed by Lubliner and co-authors [60] suitable also for brittle materials, such as bricks and stones. The failure condition is defined by the following function

$f(\sigma)=\frac{1}{1-\alpha}\left[\alpha I_{1}+\sqrt{3 J_{2}}+\beta\left\{\hat{\sigma}_{\max }\right\}-\gamma\left\{-\hat{\sigma}_{\max }\right\}\right]-\sigma_{c}$

where $I_{1}$ and $J_{2}$ are the first invariant of the isotropic stress tensor and the second invariant of the deviatoric stress tensor respectively. The dimensionless constants $\alpha, \beta$ and $\gamma$,

\begin{tabular}{lcccc}
\hline Phase & $\sigma_{c}[\mathrm{MPa}]$ & $\sigma_{t}[\mathrm{MPa}]$ & $\sigma_{b}[\mathrm{MPa}]$ & $\rho$ \\
\hline Brick & 15.41 & 1.5 & 17.0 & 0.667 \\
Mortar & 5.08 & 0.5 & 6.0 & 0.667 \\
\hline
\end{tabular}

Table 2 Mechanical characteristics of the masonry constituents: $\sigma_{c}$ uniaxial compression; $\sigma_{t}$ uniaxial tension; $\sigma_{b}$ equibiaxial compression; $\rho$ tensile to compressive second stress invariant ratio evaluated in the meridian plane.

defined as

$$
\begin{aligned}
& \alpha=\frac{\sigma_{b}-\sigma_{c}}{2 \sigma_{b}-\sigma_{c}} \\
& \beta=\frac{\sigma_{c}}{\sigma_{t}}(1-\alpha)-(1+\alpha) \\
& \gamma=\frac{3(1-\rho)}{2 \rho-1}
\end{aligned}
$$

depend on uniaxial tension and compression $\left(\sigma_{t}\right.$ and $\left.\sigma_{c}\right)$, on the equibiaxial compression $\left(\sigma_{b}\right)$ and on the ratio $(\rho)$ between the second stress invariant evaluated on the tensile meridian and on the compressive meridian at initial yield for any given value of the first invariant $I_{1}$. The term $\hat{\sigma}_{\max }$ indicates the maximum value of the principal stress tensor and the sign $\{\cdot\}$ represents the Macaulay brackets.

\subsection{Results}

The failure surfaces of the generated PUCs have been obtained by applying the overall criterion introduced in Sec. 6.1. The mechanical parameters used in the failure criteria of the constituents are reported in Tab. 2. These values are based on the experimental data taken from the literature [61] and other numerical elaborations [62].

The three basic cases of boundary conditions expressed in terms of displacements (22) have been used and the related macroscopic stress tensors $\langle\sigma\rangle_{\xi}$ superimposed by means of the three coefficients $a, b, c$ (29). The reference system and the type of boundary conditions have allowed to obtain 3D failure surfaces in the $\left(\sigma_{n} \sigma_{p} \tau\right)$ stresses space, which gives an effective representation of the orthotropic properties of masonry [63].

The superposition principle has been applied by varying $a$ and $b$ coefficients according to the following

$b=\mp|a| \pm 1 \quad a \in[-1,1]$

at fixed values of $c$. In this way several closed curves belonging to the spatial failure surface have been obtained.

The failure surfaces given by the application of the procedure to each PUC of QPT\#1 are shown in Fig. 7. In particular, Fig. 7(a) presents the traces of the surfaces in the plane $\left(\sigma_{n}, \sigma_{p}\right)$ for $\tau=0\left[\mathrm{~N} / \mathrm{mm}^{2}\right]$, while Fig. $7(\mathrm{~b})$ in the plane $\left(\sigma_{b}, \tau\right)$, where $\sigma_{b}$ is the axis of equibiaxial stresses $\left(\sigma_{b}=\sigma_{n}=\sigma_{p}\right)$. In both the graphs, the grey lines are related to the surfaces of the PUCs while the bold black line 


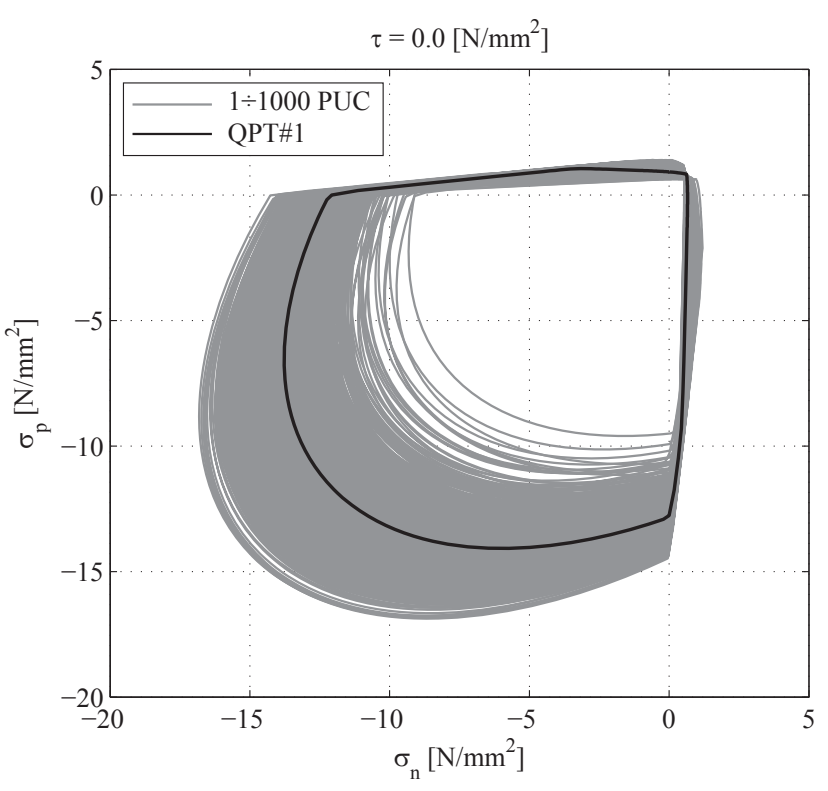

(a)

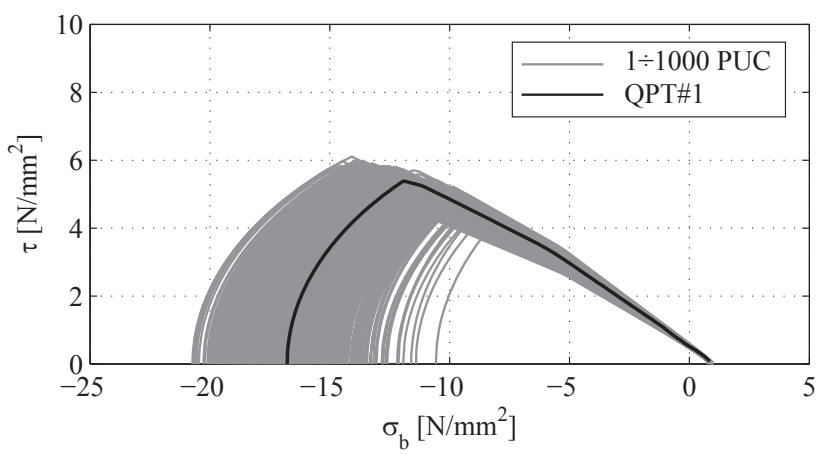

(b)

Fig. 7 QPT\#1 results. Failure surfaces for generated PUCs and QPT\#1 in the $\sigma_{n} \sigma_{p} \tau$ stresses space. (a) Section with a plane parallel to $\left(\sigma_{n}, \sigma_{p}\right)$ at $\tau=0\left[\mathrm{~N} / \mathrm{mm}^{2}\right]$. (b) Section with a $\left(\sigma_{b}, \tau\right)$ plane.

represents the domain of the homogenized continuum of the QPT\#1. For more details bout the method used to achieve such domain, the reader is invited to see [35].

As can be appreciated, the failure surfaces have the same shape although the volume enclosed, i.e. the volume of the strength domain, can be very different. In the following, the volume related to the generic PUC will be indicated with $\mathscr{V}^{\mathrm{PUC}}$, while the volume subtended by the effective failure surface of the actual QPT\#1 with $\mathscr{V}^{\text {eff }}$. The latter was derived by averaging the apparent strength domain volumes obtained by the application of natural end essential boundary conditions, i.e. $\mathscr{V}_{\sigma}^{\text {app }}$ and $\mathscr{V}_{\varepsilon}^{\text {app }}$ respectively. All these volumes have been evaluated through a numerical procedure [35]. In Fig. 8(a) the values of $\mathscr{V}^{\mathrm{PUC}}$ have been plotted in a points cloud and compared with the values of $\mathscr{V}_{\sigma}^{\text {app }}$ and $\mathscr{V}_{\varepsilon}^{\text {app }}$ (red lines). It should be noted that the SEPUC\#1, identified by the blue point, is out of bounds delimited by $\mathscr{V}_{\sigma}^{\text {app }}$ and $\mathscr{V}_{\varepsilon}^{\text {app }}$. Nevertheless, the volume difference $\Delta \mathscr{V}^{\mathrm{PUC}}=$
$\left|\mathscr{V}^{\mathrm{PUC}}-\mathscr{V}^{\mathrm{eff}}\right|$ normalized with respect to $\mathscr{V}^{\mathrm{eff}}$ could be considered contained within some percentage points. Such differences have been calculated for all the PUCs and shown in Fig. 8(b). The reliability of the results can be also observed in the graphical comparison between the failure surfaces of the SEPUC\#1 (red line) and of the QPT\#1 (black line) shown in Fig. 9.

The procedure carried outon the actual QPT\#2 has led to similar results, shown in the Appendix A.

\subsection{Remarks}

On the basis of the obtained results, it should be noted that by the mechanical point of view the definition of a Statistically Equivalent Periodic Unit Cell requires the study of a multi-objective problem. The searching for the solution of a multi-objective function does not always lead to a unique results, defined as the optimum. In such problems the optimal solution may not exists, so that is necessary to define a set of possible and acceptable cases that could be taken into account. An interesting instrument used in the multi-criteria and multi-objective optimization problems is the research of the Pareto front, which is a property of each specific problem [64]. The Pareto front consists of a set of feasible solutions, which represent the problem trade-offs shown in a useful representative manner for the decision making. In this work, the concept of the Pareto solutions is used to verify the effectiveness of the criterion used in the SEPUC definition, considering for the bi-objective function the searching for the best solution of the elastic matrix and the failure surface of the periodic cell. As observed in the previous sections, the SEPUCs analyzed for both the cases QPT\#1 and QPT\#2 minimize the normed differences with the elastic matrix evaluated for the entire wall and, at the same time, estimate the failure surface, with a volume error of the strength domain of about 6\% and 2-3\% for the QPT\#1 and QPT\#2 cases respectively.

As far as it concerns the QPT\#1, if the two objective functions, i.e. the error functions of the elastic matrices and of the strength domain volumes both normalized with respect to their effective values, are shown in a graph, the Pareto front can be obtained (Fig. 10(a)). For the clearness of explanation, Fig. 10(b) represents a zoom view of Fig. 10(a), in particular the graph area delimited by the filled rectangle. It should be noted that Pareto front is well identified and that the SEPUC\#1, indicated through the blue point, belongs to the set of Pareto optimal solutions. Regarding the solution obtained with a simple running bond pattern, indicated as SEPUC\#1-RB, the results have highlighted differences of about $4-5 \%$ of the elastic components with respect to the minor differences given by the SEPUC\#1. This aspect is more evident for the vertical elastic component $\mathrm{C} 22$, where 

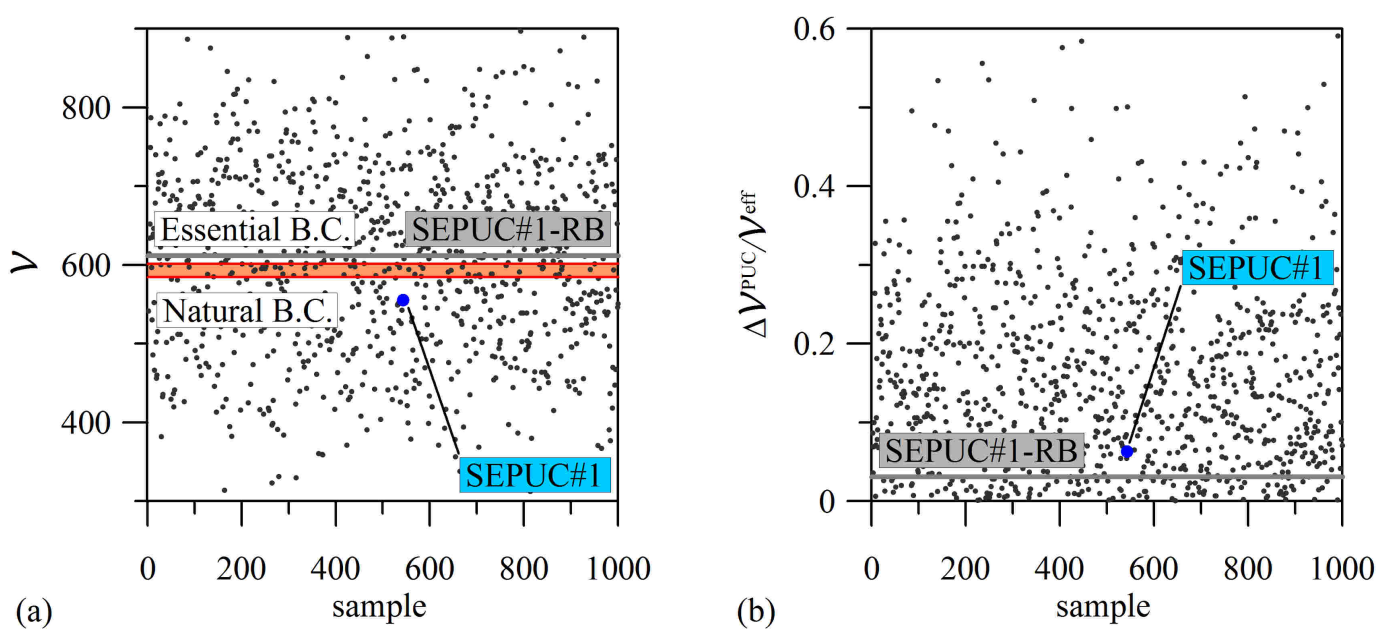

Fig. 8 QPT\#1 results. (a) Comparison between the strength domain volume evaluated for each PUC, thorugh the application of periodic boundary conditions (black points), and for the QPT\#1, through the application of essential and natural boundary conditions (red lines). (b) Error estimation of strength domain volume normalized with respect to $\mathscr{V}^{\text {eff }}$ evaluated for each PUC. The blue point identifies the SEPUC\#1 and the grey line indicates the value obtained for the SEPUC\#1-RB.

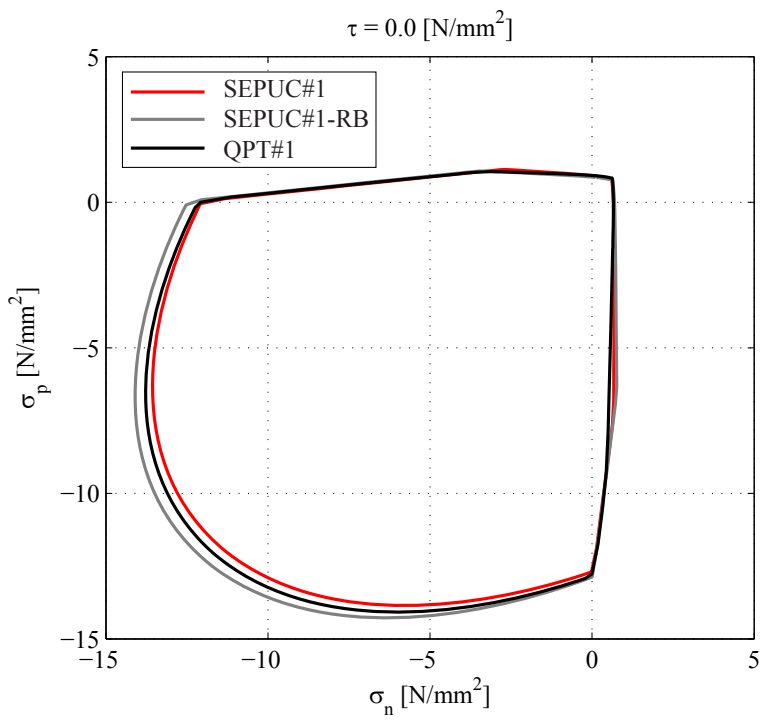

(a)

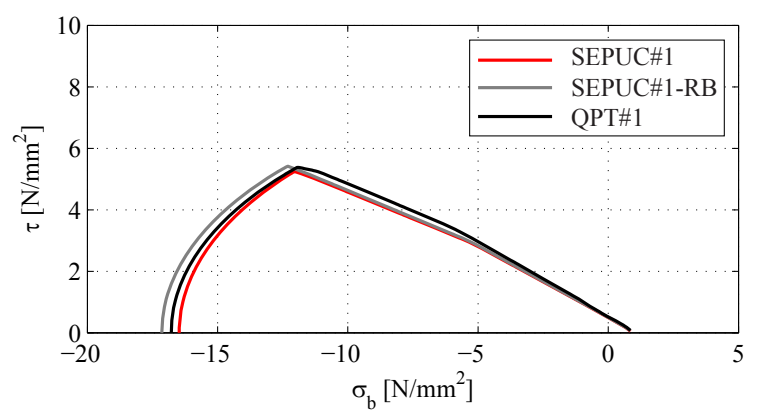

(b)

Fig. 9 QPT\#1 results. Failure surface of SEPUC\#1 (red line), SEPUC\#1-RB (grey line) and QPT\#1 (black line) in the $\left(\sigma_{n}, \sigma_{p}, \tau\right)$ stresses space. (a) Section with a plane parallel to $\left(\sigma_{n}, \sigma_{p}\right)$ at $\tau=$ $0 \mathrm{~N} / \mathrm{mm}^{2}$. (b) Section with a $\left(\sigma_{b}, \tau\right)$ plane. the bimodal distribution of the stones heights has been neglected. Even if the procedure seems to give a better result for the SEPUC\#1-RB in terms of failure surface (Fig. 9(b)), the combination between elastic and at failure results highlight that the SEPUC\#1 represents a more consistent solution (Fig. 10(b)).

The previous results have been confirmed when the quasiperiodic textures QPT\#2 is considered (see Appendix A). By using the SEPUC\#2 it is possible to obtain a very good assessment of the elastic parameters (Figg. 11-12) and an approximated solution of the failure surfaces (Figg. 13-14). In fact the SEPUC\#2 does not belong to the Pareto front (Fig. 15) but the differences are very limited.

\section{Conclusions}

In this paper a method for the estimation of the failure surface of quasi-periodic masonry has been presented. This procedure is based on the application of an approximated failure criterion, already validated on masonry material in previous work by the Authors to the Statistically Equivalent Periodic Unit Cell (SEPUC), which is related to a quasi-periodic masonry texture. In the paper two actual cases have been studied.

The definition of the SEPUC refers to a probabilistic approach based on the statistical analysis of the texture. Starting from the geometrical descriptors, a population of Periodic Unit Cells (PUCs) has been obtained. Among the generated samples, the SEPUC has been identified by a geometrical criterion, which minimizes an error function defined on the geometrical descriptors and the concentration ratio of the phases. As far as the elastic properties are concerned the SEPUC has been validated with a novel method which takes 

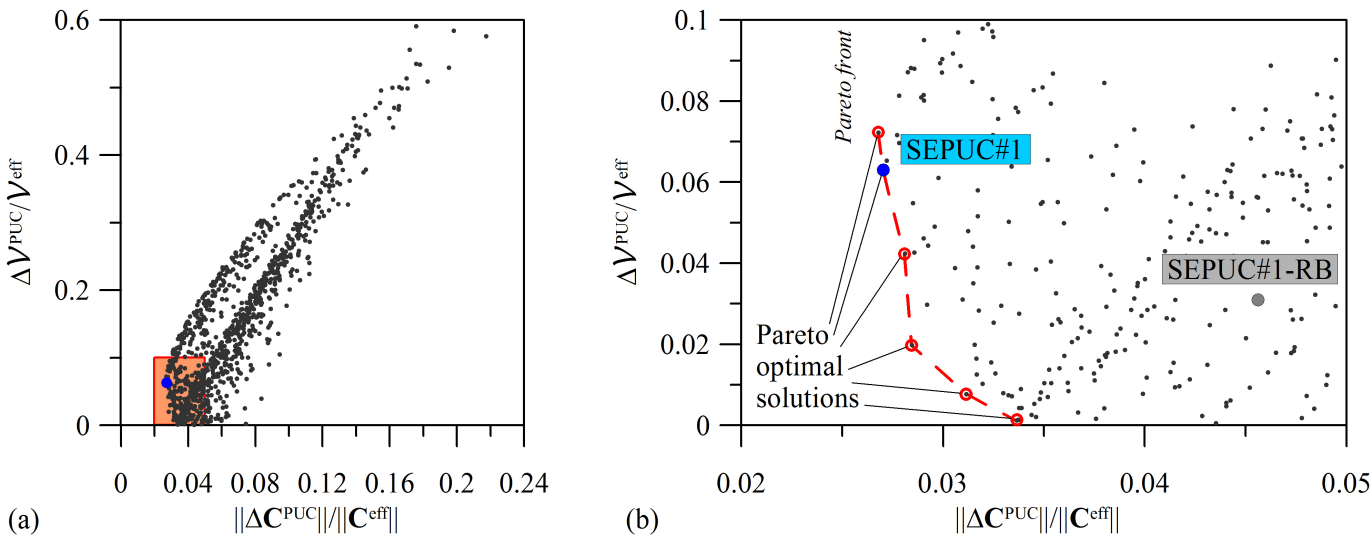

Fig. 10 QPT\#1 results. (a) Representation of the multi-objective function obtained by the analysis of the elastic properties and the failure condition of the PUCs related to QPT\#1. (b) Zoom of Fig. 10(a) (orange area) in which Pareto front is identified. The blue and the grey points identify SEPUC\#1 and SEPUC\#1-RB respectively.

into account individually the residuals of all the components of the elastic matrix.

Moreover, the validation of the statistical criterion has been extended also to the case of the failure condition. It has been noted that identification of the optimal cell has required the solution of a multi-objective function, i.e. the elastic properties and the failure condition. To this purpose the Pareto front approach has been used. According to this theory a set of optimal solutions exists, which defines the front. It has been highlighted that the SEPUC belongs, or it is very closed, to the Pareto front in both the actual cases analysed.

The obtained results assert that the SEPUC approach, only defined through geometrical analysis, allows to obtain a periodic cell representative of an actual quasi-periodic masonry texture, both for the elastic properties and the failure condition assessment of the homogenized continuum, as an optimal solution of the Pareto front in a multi-objective approach. Moreover, even if the comparison between the results obtained by the application of such homogenization methods and other methodologies based on non-linear micromechanical approaches or limit analysis could be of interest, it is worth noting that the presented procedure allows to rapidly obtain the SEPUC and related homogenized properties, which can be used for several applications, as multiscale numerical procedures.

\section{A QPT\#2: SEPUC analysis}

In this section the results obtained on the quasi-periodic texture \#2 (QPT\#2) of Fig. 2(b) are shown. In order to simplify the reading of the results, the same figures illustrated for the QPT\#1 embedded in the main text, have been reported. In particular, the Figg. 11 and 12 summarize the elastic results, while Figg. 13-14 concern with the failure surfaces. In the end, Fig. 15 combines both the elastic and at failure results in the multi-objective analysis with the study of Pareto front.
Acknowledgements The Authors gratefully acknowledge support from the Italian Ministry of Education, University and Scientic Research, within the PRIN National Grant 2015 project "Advanced mechanical modeling of new materials and structures for the solution of 2020 Horizon challenges" (Grant 2015JW9NJT).

\section{Compliance with Ethical Standards:}

Conflict of Interest: The Authors declare that they have no conflict of interest.

\section{References}

1. R. Hill. Elastic properties of reinforced solids: some theoretical principles. J. Mech. Phys. Solids, 11:357-372, 1963.

2. P. Suquet. Elements of homogenization for inelastic solid mechanics. In E. Sanchez-Palencia and A. Zaoui, editors, Homogenization Techniques for Composite Media, Lecture Notes in Physics, volume 272, pages 193-278. Springer, Berlin, 1987.

3. J.L. Teply and G.J. Dvorak. Bounds on overall instantaneous properties of elastic-plastic composites. J. Mech. Phys. Solids, 36(1):29-58, 1988.

4. C. Huet. Application of variational concepts to size effects in elastic heterogeneous bodies. J. Mech. Phys. Solids, 38(6):813-841, 1990.

5. A. Anthoine. Derivation of the in-plane elastic characteristics of masonry through homogenization theory. International Journal of Solids and Structures, 32(2):137-163, 1995.

6. A. Zucchini and P.B. Lourenço. A micro-mechanical model for the homogenisation of masonry. International Journal of Solids and Structures, 39:3233-3255, 2002.

7. M. Mistler, A. Anthoine, and C. Butenweg. In-plane and outof-plane homogenisation of masonry. Computers and Structures, 85:1321-1330, 2007.

8. A. Drougkas, P. Roca, and C. Molins. Analytical micro-modeling of masonry periodic unit cells - elastic properties. International Journal of Solids and Structures, 69-70:169-188, 2015.

9. T.J. Massart, R.H.J. Peerlings, and M.G.D. Geers. Mesoscopic modeling of failure and damage-induced anisotropy in brick masonry. European Journal of Mechanics, A/Solids, 23(5):719-735, 2004 

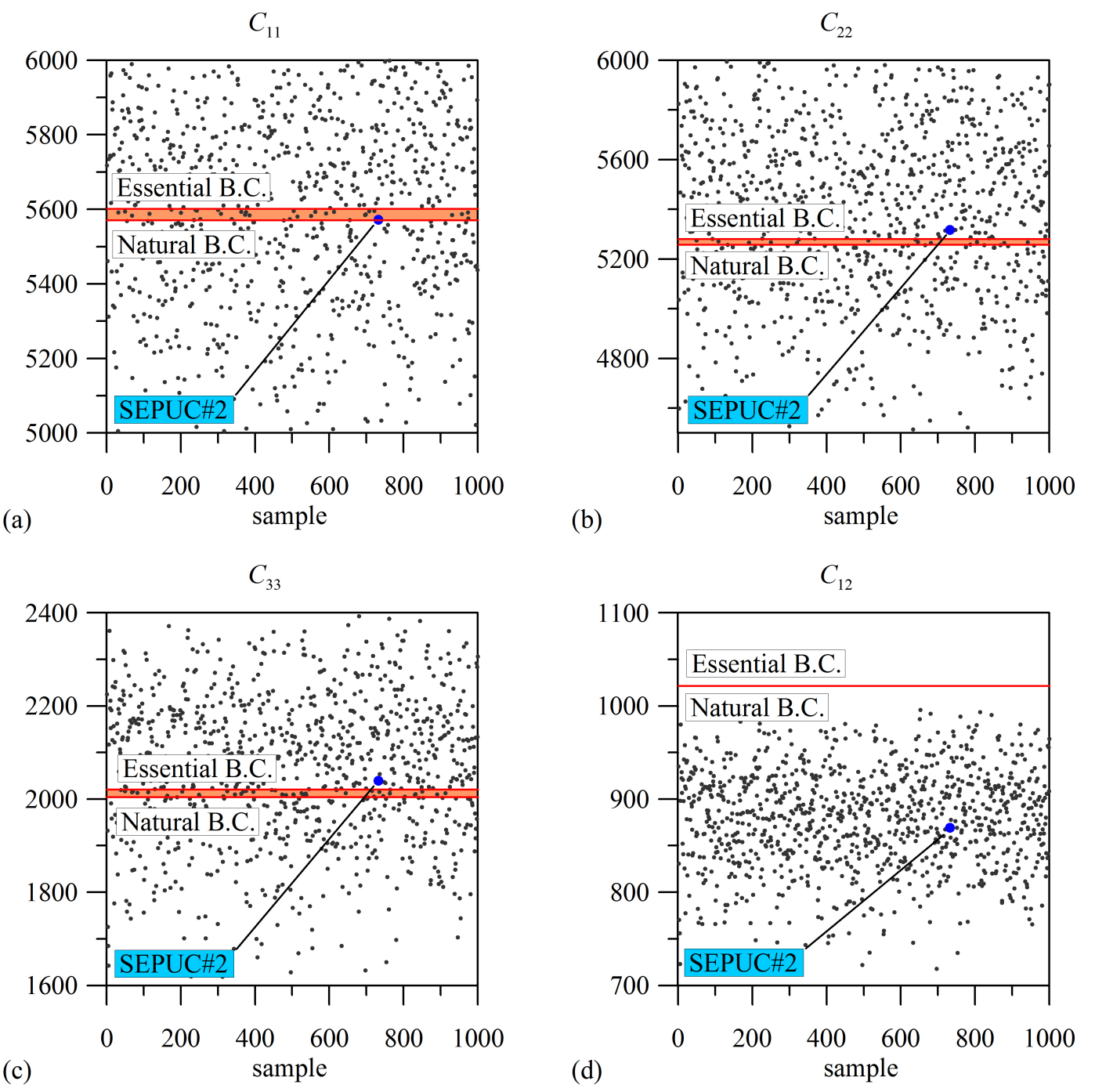

Fig. 11 QPT\#2 results. Comparison between the elastic matrix components (values in MPa) evaluated for each PUC, through the application of periodic boundary conditions (black points), and for the QPT\#2, through the application of essential and natural boundary conditions (red lines). The blue point identifies the SEPUC\#2 given by the objective function (13).

10. T.J. Massart, R.H.J. Peerlings, M.G.D. Geers, and S. Gottcheiner. Mesoscopic modeling of failure in brick masonry accounting for three-dimensional effects. Engineering Fracture Mechanics, 72(8):1238-1253, 2005 .

11. G. Milani, P. Loureno, and A. Tralli. Homogenization approach for the limit analysis of out-of-plane loaded masonry walls. Journal of Structural Engineering, 132(10):1650-1663, 2006.

12. E. Sacco. A nonlinear homogenization procedure for periodic masonry. European Journal of Mechanics, A/Solids, 28:209-222, 2009.

13. D. Addessi and E. Sacco. Nonlinear analysis of masonry panels using a kinematic enriched plane state formulation. International Journal of Solids and Structures, 90:194-214, 2016.

14. D. Addessi and E. Sacco. Enriched plane state formulation for nonlinear homogenization of in-plane masonry wall. Meccanica, 51(11):2891-2907, 2016

15. A. Drougkas, P. Roca, and C. Molins. Nonlinear micromechanical analysis of masonry periodic unit cells. International Journal of Solids and Structures, 80:193-211, 2016.

16. D. Baraldi, A. Cecchi, and A. Tralli. Continuous and discrete models for masonry like material: A critical comparative study.
European Journal of Mechanics, A/Solids, 50:39-58, 2015.

17. D. Baraldi and A. Cecchi. Discrete approaches for the nonlinear analysis of in plane loaded masonry walls: Molecular dynamic and static algorithm solutions. European Journal of Mechanics, A/Solids, 57:165-177, 2016.

18. P.B. Lourenço and J.G. Rots. Multisurface interface model for analysis of masonry structures. Journal of Engineering Mechanics, 123(7):660-668, 1997.

19. T.J. Massart, R.H.J. Peerlings, and M.G.D. Geers. An enhanced multi-scale approach for masonry wall computations with localization of damage. Int. J. Numer. Meth. Engng., 69:1022-1059, 2007.

20. S. Brasile, R. Casciaro, and G. Formica. Multilevel approach for brick masonry walls - part i: A numerical strategy for the nonlinear analysis. Comput. Methods Appl. Mech. Engrg., 196(4952):4934-4951, 2007.

21. F. Greco, L. Leonetti, R. Luciano, and P. Nevone Blasi. An adaptive multiscale strategy for the damage analysis of masonry modeled as a composite material. Composite Structures, 153:972-988, 2016. 

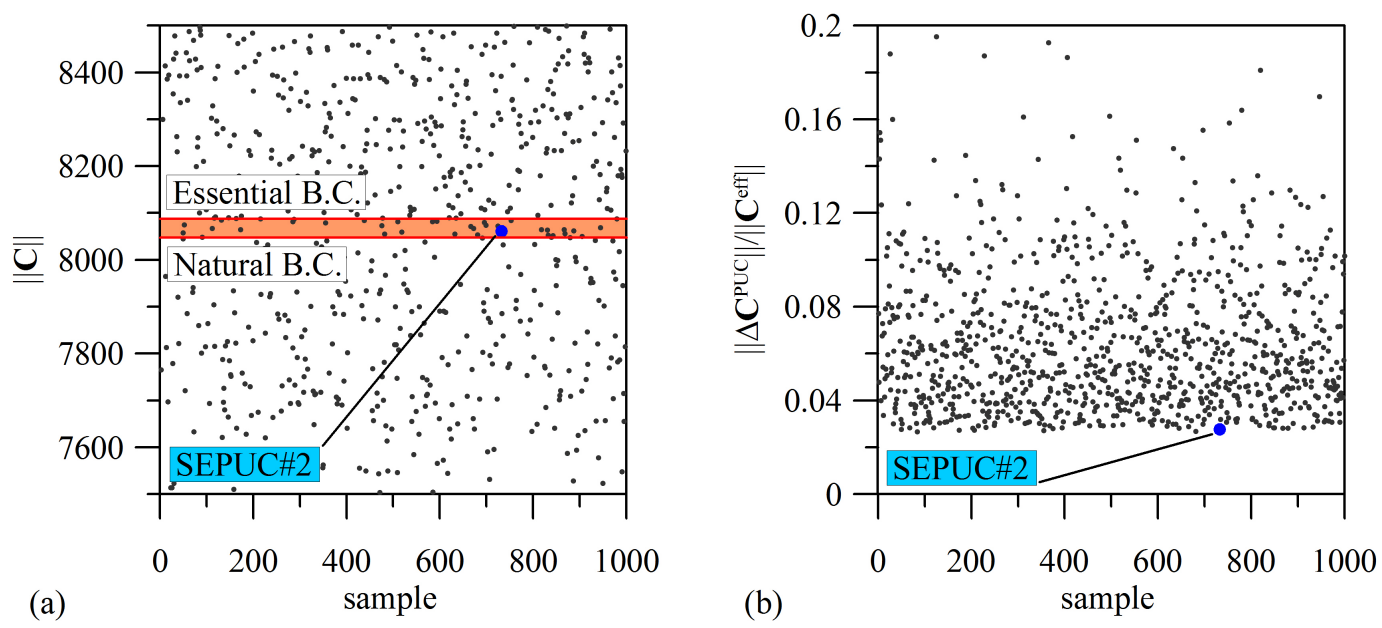

Fig. 12 QPT\#2 results. (a) Comparison between the Frobenius norm of the elastic matrix evaluated for each PUC, thorugh the application of periodic boundary conditions (black points), and for the QPT\#2, through the application of essential and natural boundary conditions (red lines). (b) Error estimation $\left\|\Delta \mathbf{C}^{\mathrm{PUC}}\right\|$ evaluated for each PUC normalized with respect to the effective elastic matrix norm $\left\|\mathbf{C}^{\text {eff }}\right\|$. The blue point identifies the SEPUC\#2.
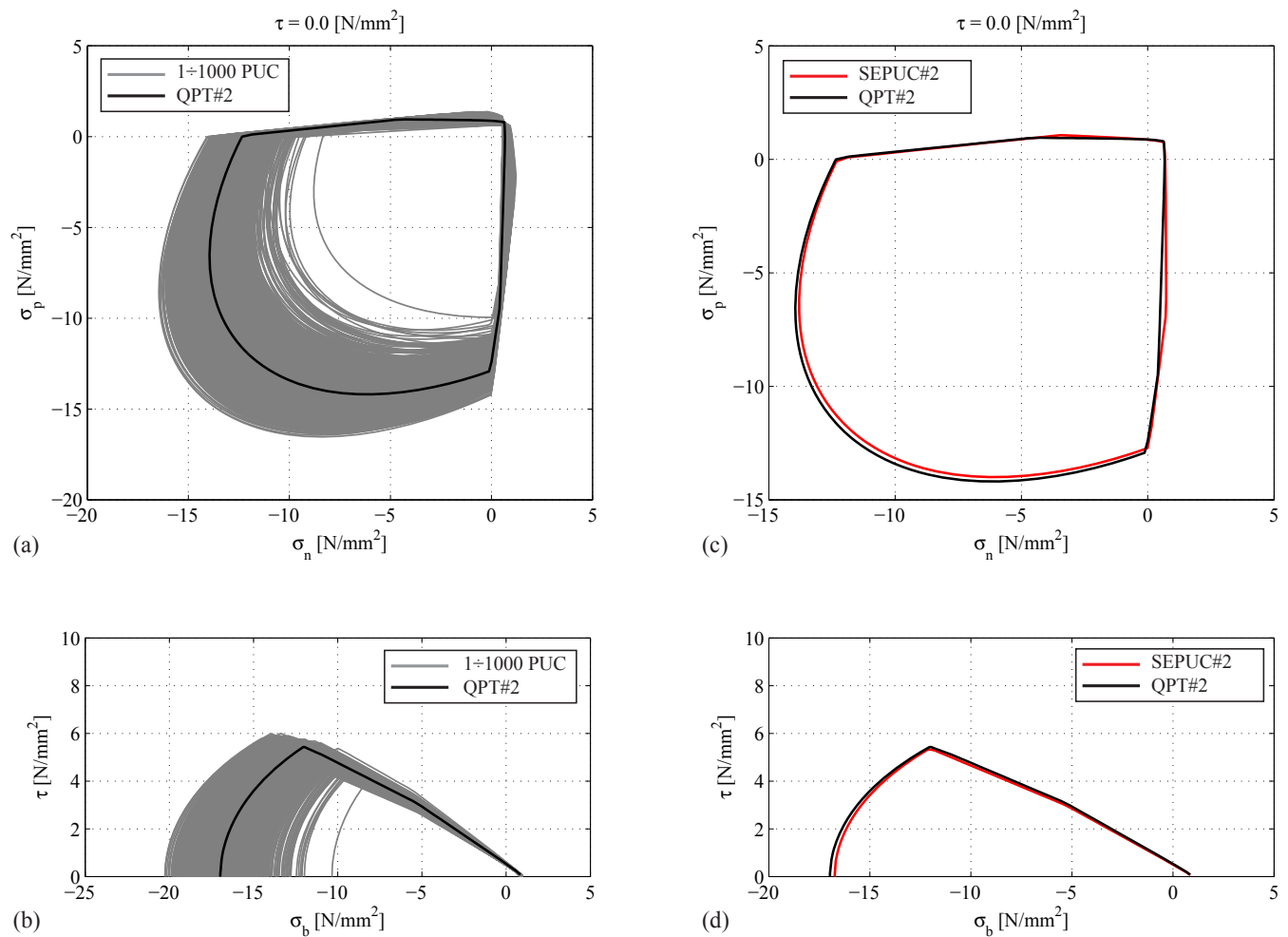

Fig. 13 QPT\#2 results. (a-b) Failure surfaces for generated PUCs and QPT\#2 in the $\sigma_{n} \sigma_{p} \tau$ stresses space: (a) section with a plane parallel to $\left(\sigma_{n}, \sigma_{p}\right)$ at $\tau=0\left[\mathrm{~N} / \mathrm{mm}^{2}\right]$; (b) section with a $\left(\sigma_{b}, \tau\right)$ plane. (c-d) Failure surfaces of SEPUC\#2 (red line) and QPT\#2 (black line) in the $\left(\sigma_{n}, \sigma_{p}, \tau\right)$ stresses space: (c) section with a plane parallel to $\left(\sigma_{n}, \sigma_{p}\right)$ at $\tau=0 \mathrm{~N} / \mathrm{mm}^{2} ;$ (d) section with a $\left(\sigma_{b}, \tau\right)$ plane.

22. P. Trovalusci and R. Masiani. Cosserat and cauchy materials as continuum models of brick masonry. Meccanica, 31:421-432, 1996.

23. P. Trovalusci and R. Masiani. Non-linear micropolar and classical continua for anisotropic discontinous materials. International Journal of Solids and Structures, 40(5):1281-1297, 2003.

24. M.L. De Bellis and D. Addessi. A cosserat based multi-scale model for masonry structures. International Journal for Multiscale Computational Engineering, 9(5):543-563, 2011.
25. Q.-C. He. Effects of size and boundary conditions on the yield strength of heterogeneous materials. J. Mech. Phys. Solids, 49:2557-2575, 2001.

26. M. Ostoja-Starzewski. Material spatial randomness: From statistical to representative volume element. Probab. Eng. Mech., 21:112-132, 2006

27. P. Trovalusci, M. Ostoja-Starzewski, M.L. De Bellis, and A. Murrali. Scale-dependent homogenization of random composites as micropolar continua. European Journal of Mechanics, A/Solids, 

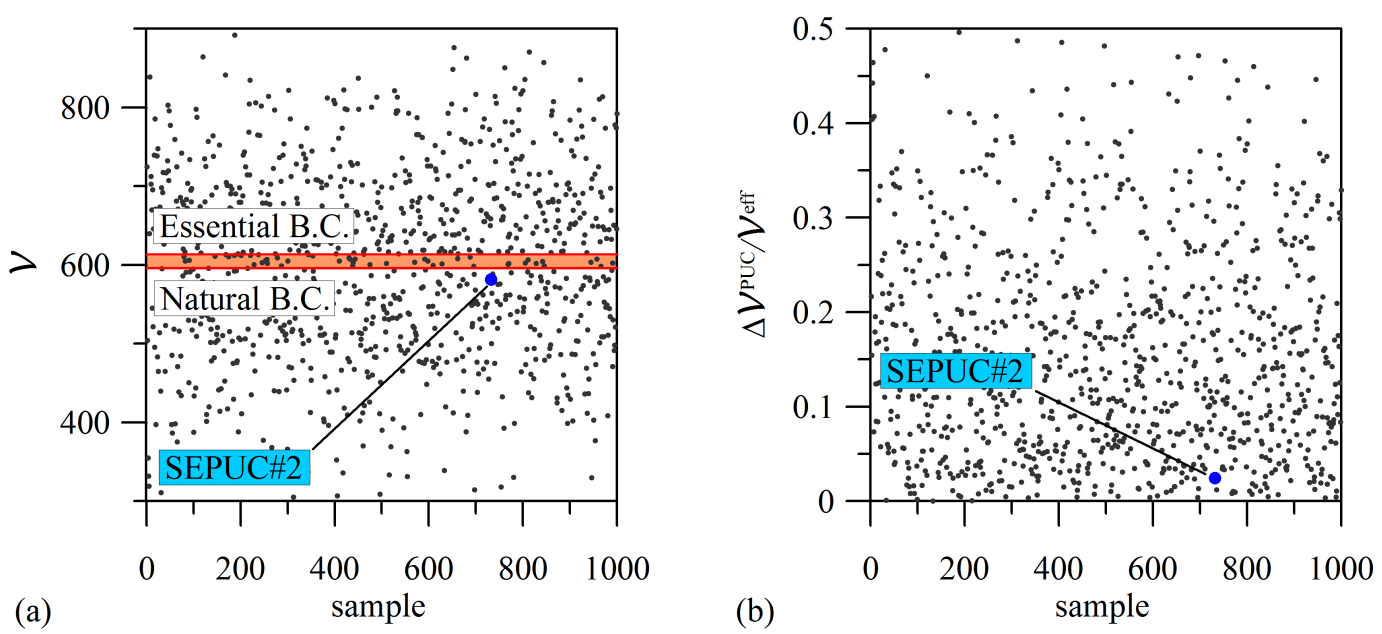

Fig. 14 QPT\#2 results. (a) Comparison between the strength domain volume evaluated for each PUC, thorugh the application of periodic boundary conditions (black points), and for the QPT\#2, through the application of essential and natural boundary conditions (red lines). (b) Error estimation of strength domain volume normalized with respect to $\mathscr{V}$ eff evaluated for each PUC. The blue point identifies SEPUC\#2.
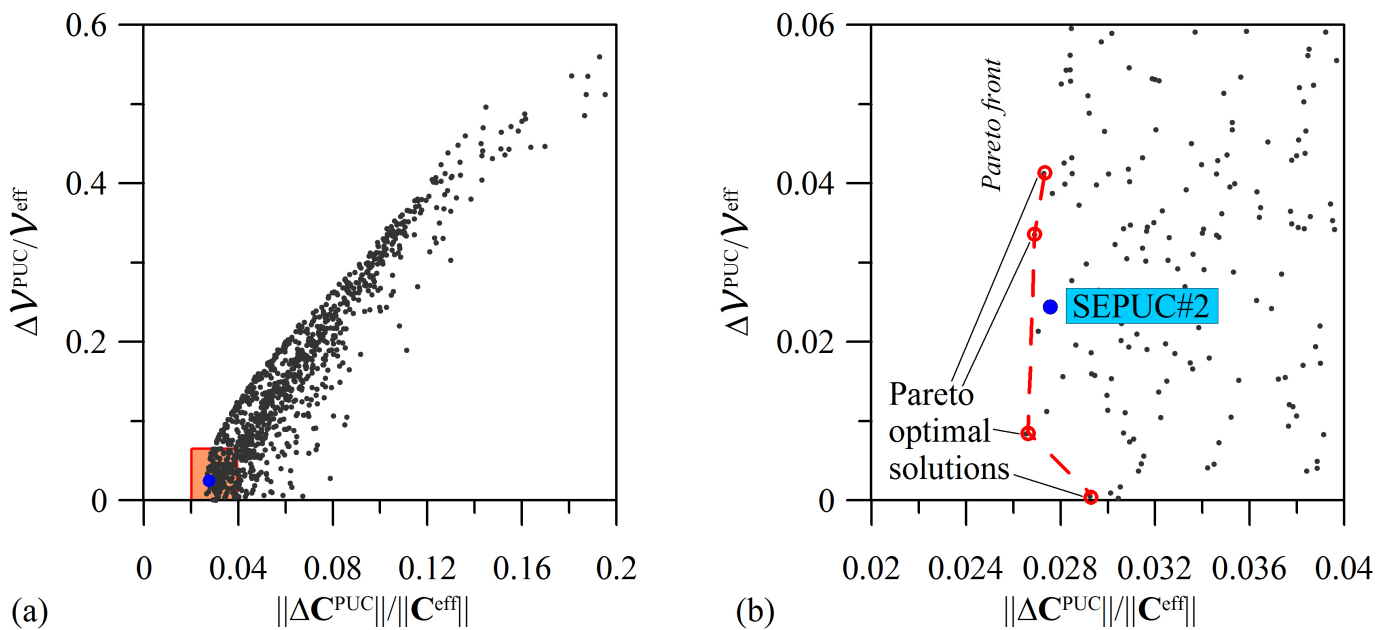

Fig. 15 QPT\#2 results. (a) Representation of the multi-objective function obtained by the analysis of the elastic properties and the failure condition of the PUCs related to QPT\#2. (b) Zoom of Fig. 10(a) (orange area) in which Pareto front is identified. The blue point identifies SEPUC\#2.

49:396-407, 2015.

28. F. Cluni and V. Gusella. Homogenization of non-periodic masonry structures. Int. J. of Solids and Structures, 41:1911-1923, 2004.

29. V. Gusella and F. Cluni. Random field and homogenization for masonry with nonperiodic microstructure. Journal of Mechanics of Materials and Structures, 1(2):357-386, 2006.

30. G. Falsone and M. Lombardo. Stochastic representation of the mechanical properties of irregular masonry structures. International Journal of Solids and Structures, 44:8600-8612, 2007.

31. A. Cecchi and K. Sab. Discrete and continuous models for in plane loaded random elastic brickwork. European Journal of Mechanics, A/Solids, 28:610-625, 2009.

32. K. Sab. Overall ultimate yield strength of a quasi-periodic masonry. C. R. Mecanique, 337:603-609, 2009.

33. G. Milani and P.B. Lourenço. Monte carlo homogenized limit analysis model for randomly assembled blocks in-plane loaded. Computational Mechanics, 46:827-849, 2010.

34. G. Milani and P.B. Lourenço. A simplified homogenized limit analysis model for randomly assembled blocks out-of-plane loaded. Computers \& Strucutres, 88:690-717, 2010.
35. N. Cavalagli, F. Cluni, and V. Gusella. Strength domain of nonperiodic masonry by homogenization in generalized plane state. European Journal of Mechanics - A/Solids, 30:113-126, 2011.

36. M. Ostoja-Starzewski. Material spatial randomness: From statistical to representative volume element. Probabilistic Engineering Mechanics, 21(2):112-132, 2006.

37. K. Sab and B. Nedjar. Periodization of random media and representative volume element size for linear composites [priodisation des milieux alatoires et dtermination de la taille du volume Imentaire reprsentatif des composites linaires]. Comptes Rendus Mecanique, 333(2):187-195, 2005.

38. S. Swaminathan, S. Ghosh, and N.J. Pagano. Statistically equivalent representative volume elements for unidirectional composite microstructures: Part i - without damage. Journal of Composite Materials, 40:583-604, 2009.

39. S. Swaminathan and S. Ghosh. Statistically equivalent representative volume elements for unidirectional composite microstructures: Part ii - with interfacial debonding. Journal of Composite Materials, 40:605-621, 2009.

40. J. Zeman and M. Šejnoha. From random microstructures to representative volume elements. Modelling and Simulation in Materials Science and Engineering, 15(4):S325-S335, 2007. 
41. D. Balzani, L. Scheunemann, D. Brands, and J. Schrder. Construction of two- and three-dimensional statistically similar rves for coupled micro-macro simulations. Computational Mechanics, 54(5):1269-1284, 2014.

42. L. Scheunemann, D. Balzani, D. Brands, and J. Schrder. Design of 3d statistically similar representative volume elements based on minkowski functionals. Mechanics of Materials, 90:185-201, 2015.

43. P. Trovalusci, M.L. De Bellis, and M. Ostoja-Starzewski. A statistically-based homogenization approach for particle random composites as micropolar continua. Advanced Structured Materials, 42:425-441, 2016.

44. S.M.J. Spence, M. Gioffrè, and M.D. Grigoriu. Probabilistic models and simulation of irregular masonry walls. Journal of Engineering Mechanics, 134(9):750-762, 2008.

45. M. Lombardo, J. Zeman, M. Šejnoha, and G. Falsone. Stochastic modeling of chaotic masonry via mesostructural characterization. International Journal for Multiscale Computational Engineering, 7(2):171-185, 2009

46. J. Šejnoha, M. Šejnoha, J. Zeman, J. Sykora, and J. Vorel. Mesoscopic study on historic masonry. Structural Engineering and Mechanics, 30(1):99-117, 2008.

47. J. Zeman, J. Novk, M. Šejnoha, and J. Šejnoha. Pragmatic multiscale and multi-physics analysis of charles bridge in prague. Engineering Structures, 30(11):3365-3376, 2008.

48. N. Cavalagli, F. Cluni, and V. Gusella. Evaluation of a statistically equivalent periodic unit cell for a quasi-periodic masonry. International Journal of Solids and Structures, 50:4226-4240, 2013.

49. B. Riveiro, P.B. Loureno, D.V. Oliveira, H. Gonzlez-Jorge, and P. Arias. Automatic morphologic analysis of quasi-periodic masonry walls from lidar. Computer-Aided Civil and Infrastructure Engineering, 31(4):305-319, 2016.

50. A.W. Page. Finite element model for masonry. Journal of Structural Division ASCE, 104(ST8):1267-1285, 1978.

51. P. de Buhan and G. de Felice. A homogenization approach to the ultimate strength of brick masonry. Journal of the Mechanics and Physics of Solids, 45(7):1085-1104, 1997.

52. R. Luciano and E. Sacco. A damage model for masonry structures. European Journal of Mechanics, A/Solids, 17(2):285-303, 1998.

53. G. Milani, P.B. Lourenço, and A. Tralli. Homogenised limit analysis of masonry walls, part i: Failure surfaces. Computers and Structures, 84(3-4):166-180, 2006.

54. A. Caporale, F. Parisi, D. Asprone, R. Luciano, and A. Prota. Critical surfaces for adobe masonry: Micromechanical approach. Composites Part B: Engineering, 56:790-796, 2014.

55. G. Milani and A. Taliercio. In-plane failure surfaces for masonry with joints of finite thickness estimated by a method of cells-type approach. Computers and Structures, 150:34-51, 2015.

56. A.H.-D. Cheng. On generalized plane strain poroelasticity. Int. J. Rock. Min. Sci., 35(2):183-193, 1998.

57. A. Anthoine. Homogeneization of periodic masonry: plane stress, generalized plane strain or 3d modelling? Communications in $\mathrm{Nu}$ merical Methods in Engineering, 13:319-326, 1997.

58. S.G. Lekhnitskii. Theory of Elasticity of an Anisotropic Elastic Body. Mir Publishers, Moskow, USSR, 1981.

59. G.J. Dvorak and Y.A. Bahei-El-Din. A bimodal plasticity theory of fibrous composite materials. Acta Mechanica, 69:219-241, 1987.

60. J. Lubliner, J. Oliver, S. Oller, and E. Oñate. A plastic-damage model for concrete. International Journal of Solids and Structures, 25(3):229-326, 1989.

61. A.W. Page. The biaxial compressive strength of brick masonry. Proceeding of the Institution of Civil Engineers London, Part 2, 71:893-906, 1981.

62. B. Shieh-Beygi and S. Pietruszczak. Numerical analysis of structural masonry: mesoscale approach. Computers and Structures, 86:1958-1973, 2008.
63. M. Dhanasekar, A.W. Page, and P.W. Kleeman. Failure of brick masonry under biaxial stresses. Proceedings of the Institution of Civil Engineers (London), 79(pt 2):295-313, 1985.

64. G. Chiandussi, M. Codegone, S. Ferrero, and F.E. Varesio. Comparison of multi-objective optimization methodologies for engineering applications. Computers and Mathematics with Applications, 63(5):912-942, 2012. 